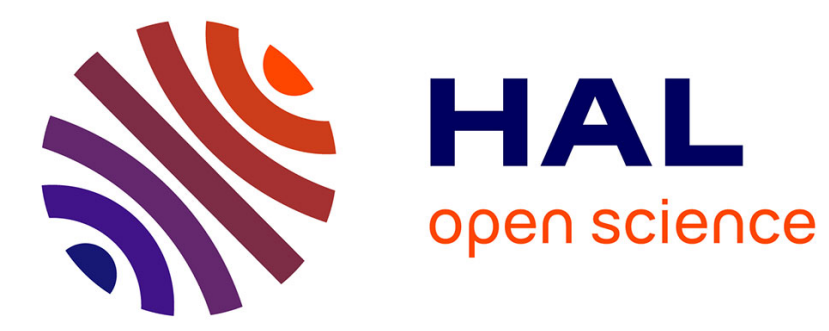

\title{
Algebras with involution that become hyperbolic over the fonction field of a conic
}

\author{
Anne Quéguiner-Mathieu, Jean-Pierre Tignol
}

\section{To cite this version:}

Anne Quéguiner-Mathieu, Jean-Pierre Tignol. Algebras with involution that become hyperbolic over the fonction field of a conic. 2008. hal-00282238

\section{HAL Id: hal-00282238 \\ https://hal.science/hal-00282238}

Preprint submitted on 26 May 2008

HAL is a multi-disciplinary open access archive for the deposit and dissemination of scientific research documents, whether they are published or not. The documents may come from teaching and research institutions in France or abroad, or from public or private research centers.
L'archive ouverte pluridisciplinaire HAL, est destinée au dépôt et à la diffusion de documents scientifiques de niveau recherche, publiés ou non, émanant des établissements d'enseignement et de recherche français ou étrangers, des laboratoires publics ou privés. 


\title{
ALGEBRAS WITH INVOLUTION THAT BECOME HYPERBOLIC OVER THE FUNCTION FIELD OF A CONIC
}

\author{
ANNE QUÉGUINER-MATHIEU AND JEAN-PIERRE TIGNOL
}

\begin{abstract}
We study central simple algebras with involution of the first kind that become hyperbolic over the function field of the conic associated to a given quaternion algebra $Q$. We classify these algebras in degree 4 and give an example of such a division algebra with orthogonal involution of degree 8 that does not contain $(Q,-)$, even though it contains $Q$ and is totally decomposable into a tensor product of quaternion algebras.
\end{abstract}

Given two central simple algebras with involution $(A, \sigma)$ and $(B, \tau)$ over a field $F$, we say that $(A, \sigma)$ contains $(B, \tau)$ if $A$ contains a $\sigma$-stable subalgebra isomorphic to $B$ over which the involution induced by $\sigma$ is conjugate to $\tau$. By the double centralizer theorem [11, (1.5)], this is also equivalent to saying that $(A, \sigma)$ is isomorphic to a tensor product $(A, \sigma) \simeq(B, \tau) \otimes(C, \gamma)$ for some central simple algebra with involution $(C, \gamma)$ over $F$.

Let $(Q,-)$ be a quaternion division algebra over $F$, endowed with its canonical involution. We denote by $F_{Q}$ the function field of the associated conic, which is the Severi-Brauer variety of $Q$. Since - is of symplectic type, it becomes hyperbolic over any field that splits $Q$, hence in particular over $F_{Q}$. From this, one may easily deduce that any $(A, \sigma)$ that contains $(Q,-)$ becomes hyperbolic over $F_{Q}$. The main theme of this paper is to investigate the reverse implication. In the case where $A$ is split and $\sigma$ is anisotropic, it is an easy consequence of the Cassels-Pfister subform theorem in the algebraic theory of quadratic forms that the converse holds, see Proposition 2.1. When $A$ is not split, the problem is much more delicate, and comparable to the characterization of quadratic forms that become isotropic over $F_{Q}$, which was studied by Hoffmann, Lewis, and Van Geel [8], [9], [1]]. Using an example from [10] of a 7 -dimensional quadratic form over a suitable field $F$ that becomes isotropic over $F_{Q}$, we construct in $\$$ 河 a division algebra $A$ of degree 8 with an orthogonal involution $\sigma$ such that $(A, \sigma)$ becomes hyperbolic over $F_{Q}$ but does not contain $(Q,-)$, even though $A$ contains $Q$ and $(A, \sigma)$ decomposes into a tensor product of quaternion algebras with involution. This situation does not occur in lower degrees.

Algebras with involution that become split hyperbolic over $F_{Q}$ are considered in \$2. It is shown in Proposition 2.3 that their anisotropic kernel contains $(Q,-)$ (if it is not trivial). This applies in particular to algebras of degree $2 m$ with $m$ odd, see Corollary 2.4. The case of algebras of degree 4 is completely elucidated in \$3 invariant of degree 3 due to Knus-Lam-Shapiro-Tignol for symplectic involutions. In the symplectic case, we classify the algebras of degree 4 that become hyperbolic

The second author gratefully acknowledges the hospitality of Université Paris 13, where part of the work leading to this paper was carried out. He was supported in part by the F.R.S.-FNRS. 
over $F_{Q}$ but do not contain $(Q,-)$, see Theorem 3.6. We show in 8 迎 that our result is equivalent to the Hoffmann-Lewis-Van Geel classification of 5-dimensional quadratic forms that become isotropic over $F_{Q}$ without containing a Pfister neighbour of the norm form of $Q$, see Corollary 4.2. Orthogonal involutions on algebras of degree 8 are considered in $\S \S$ 同 and 6 , using triality. In $\S$ 国 we relate tensor products of quaternion algebras to quadratic forms of dimension 8 with trivial discriminant. The algebras with involution that do not decompose into tensor products of quaternion algebras with involution and become hyperbolic over $F_{Q}$ are determined in $\S 6$, see Theorem 6.3. They are isotropic, and their anisotropic kernel contains $(Q,-)$. Finally, we use Laurent power series in $\S$ to construct algebras with involution of large degree that do not contain $(Q,-)$.

\section{Notations AND PRELIMINARY OBSERVATIONS}

We work over a base field $F$ of characteristic different from 2, and only consider algebras with involution of the first kind. We refer the reader to [11] and to [12] for background information on central simple algebras with involution and on quadratic forms. However, we depart from the notation in 12] by using $\left\langle\left\langle a_{1}, \ldots, a_{n}\right\rangle\right\rangle$ to denote the $n$-fold Pfister form $\otimes_{i=1}^{n}\left\langle 1,-a_{i}\right\rangle$.

If $h: V \times V \rightarrow D$ is a regular hermitian or skew-hermitian form on a finitedimensional vector space $V$ over a division algebra $D$, we denote by $\operatorname{ad}_{h}$ the involution on $\operatorname{End}_{D}(V)$ that is adjoint to $h$. In the particular case where $D$ is split and $h$ is the polar form of a quadratic form $q$, we also denote $\operatorname{ad}_{q}$ for $\operatorname{ad}_{h}$. Following Becher [ [1, for any $n$-dimensional quadratic form $q$ over $F$, we denote by $\operatorname{Ad}_{q}$ the split orthogonal algebra with involution $\left(M_{n}(F), \operatorname{ad}_{q}\right)$.

Recall that a central simple $F$-algebra with involution $(A, \sigma)$ is hyperbolic if and only if it is isomorphic to $\left(\operatorname{End}_{D} V, \operatorname{ad}_{h}\right)$ for some hyperbolic hermitian or skewhermitian form $h$ on a vector space $V$ over a division algebra $D$. In particular, $A$ admits a hyperbolic involution if and only if the index of $A$ divides $\frac{1}{2} \operatorname{deg}(A)$. If so, then $A$ admits up to conjugation a unique orthogonal hyperbolic involution, and a unique symplectic one; see [2] or [11, 6.B].

For any central simple $F$-algebra with involution $(A, \sigma)$ and any field extension $K / F$, we let $(A, \sigma)_{K}=\left(A \otimes_{F} K, \sigma \otimes \mathrm{Id}\right)$. As pointed out in the introduction, we shall be mostly interested in the special case where $K=F_{Q}$ is the function field of the conic associated to a quaternion algebra $Q$, i.e. its Severi-Brauer variety. Throughout the paper, we fix the notation

$$
Q=(a, b)_{F}
$$

with $a, b \in F^{\times}$, and we assume $Q$ is not split. We may then identify $F_{Q}$ with the quadratic extension $F\left(\sqrt{a t^{2}+b}\right)$ of $F(t)$, where $t$ is an indeterminate. For any central simple $F$-algebra $A$, we let $A(t)=A \otimes_{F} F(t)$ and $A[t]=A \otimes_{F} F[t]$.

Proposition 1.1. Let $(A, \sigma)$ be a central simple $F$-algebra with involution. Assume $\sigma$ is anisotropic. The following conditions are equivalent:

(a) $(A, \sigma)_{F_{Q}}$ is hyperbolic;

(b) $A(t)$ contains an element $y$ satisfying

$$
\sigma(y)=-y \quad \text { and } \quad y^{2}=a t^{2}+b .
$$

If conditions (a) and (b) hold, then $A[t]$ contains an element $y_{0}$ such that

$$
\sigma\left(y_{0}\right) y_{0}=-\left(a t^{2}+b\right) \text {. }
$$


Moreover, the following conditions are equivalent:

(a') $(A, \sigma)$ contains $(Q,-)$;

(b') $A[t]$ contains an element $y$ satisfying (11).

Proof. The equivalence of (a) and (b) readily follows from the description of anisotropic involutions that become hyperbolic over a quadratic extension in [2, 3.3]. If $y \in A(t)$ satisfies (1), then the version of the Cassels-Pfister theorem for algebras with involution in 19] yields an element $u \in A(t)$ such that $\sigma(u) u=1$ and $u y \in A[t]$. Then $y_{0}=u y$ satisfies (2).

If (a') holds, then $A$ contains two skew-symmetric elements $i, j$ such that $i^{2}=a$, $j^{2}=b$, and $j i=-i j$. Then $y=i t+j \in A[t]$ satisfies (11), so (b') holds. Conversely, suppose (b') holds and let $y \in A[t]$ satisfy (11). We then have $\sigma(y) y=-\left(a t^{2}+b\right)$, hence the degree of $y$ is 1 since $\sigma$ is anisotropic. Thus, $y=\lambda t+\mu$ for some $\lambda, \mu \in A$. It follows from (1) that $\lambda$ and $\mu$ are skew-symmetric and satisfy $\lambda^{2}=a, \mu^{2}=b$, and $\mu \lambda=-\lambda \mu$, hence they generate a $\sigma$-stable subalgebra of $(A, \sigma)$ isomorphic to $(Q,-)$.

Proposition 1.2. Let $(A, \sigma)$ be a central simple F-algebra with involution. Assume $A$ is division. If $(A, \sigma)_{F_{Q}}$ is hyperbolic, then $A$ contains $Q$ and $A[t]$ contains an element $y_{1}$ such that $y_{1}^{2}=a t^{2}+b$.

Proof. Since $(A, \sigma)_{F_{Q}}$ is hyperbolic, the algebra $A_{F_{Q}}$ is not division, hence Merkurjev's index reduction theorem [13, Th. 1] shows that $A$ contains $Q$, hence also two elements $i, j$ such that $i^{2}=a, j^{2}=b$, and $j i=-i j$. Then $y_{1}=i t+b$ satisfies $y_{1}^{2}=a t^{2}+b$.

Thus, the algebra with involution $(A, \sigma)$ in Theorem 5.2 below is such that $A(t)$ contains an element $y$ satisfying (11), and $A[t]$ contains elements $y_{0}, y_{1}$ satisfying

$$
\sigma\left(y_{0}\right) y_{0}=-\left(a t^{2}+b\right), \quad y_{1}^{2}=a t^{2}+b
$$

but no element satisfying both equations.

For the rest of this section, we focus on the case where $\sigma$ is orthogonal. We then have a discriminant $\operatorname{disc} \sigma \in F^{\times} / F^{\times 2}$ and a Clifford algebra $C(A, \sigma)$, see [11. $\S \S 7,8]$. Recall from [11, (8.25)] that the center of $C(A, \sigma)$ is the quadratic étale $F$-algebra obtained by adjoining a square root of $\operatorname{disc} \sigma$. Therefore, if $\operatorname{disc} \sigma=1$ the algebra $C(A, \sigma)$ decomposes into a direct product of two components, which are central simple $F$-algebras,

$$
C(A, \sigma) \simeq C_{+}(A, \sigma) \times C_{-}(A, \sigma) .
$$

If $\operatorname{deg} A \equiv 0 \bmod 4$, then the canonical involution $\underline{\sigma}$ on $C(A, \sigma)$ restricts to involutions $\sigma_{+}$and $\sigma_{-}$on $C_{+}(A, \sigma)$ and $C_{-}(A, \sigma)$. Moreover, the tensor product $C_{+}(A, \sigma) \otimes_{F} C_{-}(A, \sigma)$ is Brauer-equivalent to $A$, see [11, (8.12), (9.14)].

Proposition 1.3. Let $(A, \sigma)$ be a central simple F-algebra with orthogonal involution and $\operatorname{deg} A \equiv 0 \bmod 4$. If $(A, \sigma)_{F_{Q}}$ is hyperbolic, then $\operatorname{disc} \sigma=1$ and at least one of $\left(C_{+}(A, \sigma), \sigma_{+}\right),\left(C_{-}(A, \sigma), \sigma_{-}\right)$is split and isotropic.

Proof. Since $\sigma_{F_{Q}}$ is hyperbolic, disc $\sigma$ is a square in $F_{Q}$, hence also in $F$ since $F$ is quadratically closed in $F_{Q}$. By $[11$, (8.31)], the Clifford algebra of any hyperbolic involution has a split component; therefore $C_{+}(A, \sigma)_{F_{Q}}$ or $C_{-}(A, \sigma)$ is split. The corresponding involution $\sigma_{ \pm}$is isotropic over $F_{Q}$ by the main theorem in [6]. 
Since the Brauer group kernel of the scalar extension map from $F$ to $F_{Q}$ is $\{0,[Q]\}$, the description of algebras $(A, \sigma)$ with $\sigma$ orthogonal and $\operatorname{deg} A \equiv 0 \bmod 4$ such that $(A, \sigma)_{F_{Q}}$ is hyperbolic falls into two cases:

Case 1: $\left\{\left[C_{+}(A, \sigma)\right],\left[C_{-}(A, \sigma)\right]\right\}=\{0,[A]\}$

Case 2: $\left\{\left[C_{+}(A, \sigma)\right],\left[C_{-}(A, \sigma)\right]\right\}=\left\{[Q],\left[A \otimes_{F} Q\right]\right\}$.

Following Garibaldi's definition in [7], case 1 is when $(A, \sigma) \in I^{3}$. Note that these two cases are not exclusive: they intersect if and only if $A$ is Brauer-equivalent to $Q$; then $(A, \sigma)$ becomes split hyperbolic over $F_{Q}$. This case is easily dealt with in \$2. If $\operatorname{deg} A=4$, the first case arises if and only if $(A, \sigma)$ is hyperbolic, and the second case if and only if $(A, \sigma)$ contains $(Q,-)$, see $\$ 3.1$. For algebras of degree 8 , case 1 is when $(A, \sigma)$ is totally decomposable, see $\S 5$; case 2 is considered in $\S 6$.

\section{Algebras with involution that Become split hyperbolic over $F_{Q}$}

Since $F_{Q}$ is the function field of the Severi-Brauer variety of $Q$, the Brauer group kernel of the scalar extension from $F$ to $F_{Q}$ is $\{0,[Q]\}$. Therefore, if $(A, \sigma)$ is a central simple $F$-algebra with involution that becomes split hyperbolic over $F_{Q}$, then either $A$ is split or $A$ is Brauer-equivalent to $Q$. We consider each case separately.

Proposition 2.1. Let $(A, \sigma)$ be a split central simple $F$-algebra with involution.

(1) If $\sigma$ is symplectic, then it is hyperbolic. In this case, $(A, \sigma)$ contains $(Q,-)$ if and only if $\operatorname{deg} A \equiv 0 \bmod 4$.

(2) If $\sigma$ is orthogonal, then $(A, \sigma) \simeq \operatorname{Ad}_{q}$ for some quadratic form $q$. We have $\left(\operatorname{Ad}_{q}\right)_{F_{Q}}$ hyperbolic if and only if the anisotropic kernel of $q$ is a multiple of the norm form $n_{Q}$. Assuming this condition holds, $\operatorname{Ad}_{q}$ contains $(Q,-)$ if and only if the Witt index of $q$ is a multiple of 4.

Proof. (1) Since every alternating form on an $F$-vector space is hyperbolic, it follows that every split algebra with hyperbolic involution is hyperbolic. If $\rho$ is an orthogonal involution on $Q$, then $(Q,-) \otimes(Q, \rho)$ is a split algebra of degree 4 with symplectic involution. If $\operatorname{deg} A=4 m$, we have

$$
(A, \sigma) \simeq(Q,-) \otimes(Q, \rho) \otimes \operatorname{Ad}_{q}
$$

for any quadratic form $q$ of dimension $m$ since all the hyperbolic involutions on $A$ are conjugate. Conversely, if $A$ contains $Q$, then the centralizer of $Q$ in $A$ is Brauer-equivalent to $Q$, hence of even degree. Therefore, $\operatorname{deg} A \equiv 0 \bmod 4$.

(2) By definition, $\left(\mathrm{Ad}_{q}\right)_{F_{Q}}$ is hyperbolic if and only if $q_{F_{Q}}$ is hyperbolic, hence the first statement follows from [12, $\mathrm{X}(4.11)]$. Let $n_{Q} \otimes q_{0}$ be the anisotropic kernel of $q$. If the Witt index of $q$ is $4 m$, then denoting by $\mathbb{H}$ the hyperbolic plane over $F$ we have

$$
q \simeq n_{Q} \otimes\left(q_{0} \perp m \mathbb{H}\right) .
$$

Since $\operatorname{Ad}_{n_{Q}} \simeq(Q,-) \otimes(Q,-)$ (see [11, (11.1)]) it follows that

$$
\operatorname{Ad}_{q} \simeq(Q,-) \otimes(Q,-) \otimes \operatorname{Ad}_{q_{0} \perp m \mathbb{H}},
$$

so $\operatorname{Ad}_{q}$ contains $(Q,-)$. Conversely, assume $\operatorname{Ad}_{q}$ contains $(Q,-)$ and let $\left(A_{1}, \sigma_{1}\right)$ be the centralizer of $(Q,-)$ in $(A, \sigma)$. Then

$$
\operatorname{Ad}_{q} \simeq(Q,-) \otimes\left(A_{1}, \sigma_{1}\right)
$$


hence $\sigma_{1}$ is symplectic and $A_{1}$ is Brauer-equivalent to $Q$. Therefore, there is a hermitian $Q$-form $h_{1}$ such that $\sigma_{1} \simeq \operatorname{ad}_{h_{1}}$. The anisotropic kernel of $h_{1}$ has a diagonalization $\left\langle a_{1}, \ldots, a_{r}\right\rangle_{Q}$ with $a_{1}, \ldots, a_{r} \in F^{\times}$; letting $m$ be the Witt index of $h_{1}$, we have

Consider the following quadratic form over $F$ :

$$
h_{1} \simeq\left\langle a_{1}, \ldots, a_{r}\right\rangle_{Q} \perp m \mathbb{H}_{Q} .
$$

$$
q_{1}=\left\langle a_{1}, \ldots, a_{r}\right\rangle \perp m \mathbb{H} \text {. }
$$

Then $\left(A_{1}, \sigma_{1}\right) \simeq(Q,-) \otimes \operatorname{Ad}_{q_{1}}$, hence $\operatorname{Ad}_{q} \simeq \operatorname{Ad}_{n_{Q} \otimes q_{1}}$, and therefore $q$ is isometric to $n_{Q} \otimes q_{1}$ up to a scalar factor. Now,

$$
n_{Q} \otimes q_{1}=n_{Q} \otimes\left\langle a_{1}, \ldots, a_{r}\right\rangle \perp m\left(n_{Q} \otimes \mathbb{H}\right)=n_{Q} \otimes\left\langle a_{1}, \ldots, a_{r}\right\rangle \perp 4 m \mathbb{H},
$$

and $n_{Q} \otimes\left\langle a_{1}, \ldots, a_{r}\right\rangle$ is anisotropic since $\left\langle a_{1}, \ldots, a_{r}\right\rangle_{Q}$ is anisotropic (see 16. Ch. 10, Th. 1.1]). Therefore, the Witt index of $q$ is $4 m$.

For algebras that are Brauer-equivalent to $Q$, the result is as follows:

Proposition 2.2. Let $(A, \sigma)$ be a central simple F-algebra with involution such that $A$ is Brauer-equivalent to $Q$.

(1) If $\sigma$ is symplectic, then $(A, \sigma)$ contains $(Q,-)$ and $(A, \sigma)_{F_{Q}}$ is hyperbolic.

(2) If $\sigma$ is orthogonal and $(A, \sigma)_{F_{Q}}$ is hyperbolic, then $(A, \sigma)$ is hyperbolic and contains $(Q,-)$.

Proof. (1) It was already observed in the proof of Proposition 2.1(2) that every central simple algebra with symplectic involution that is Brauer-equivalent to $Q$ contains $(Q,-)$. Therefore, every such algebra becomes hyperbolic over $F_{Q}$.

(2) The first statement follows from the injectivity of the scalar extension map $W^{-}(Q,-) \rightarrow W\left(F_{Q}\right)$ proved independently in [5] and [14. If $(A, \sigma)$ is hyperbolic, then $A=M_{r}(F) \otimes Q$ for some even integer $r$, and $\sigma$ is conjugate to $\tau \otimes-$ for any symplectic (hyperbolic) involution $\tau$ on $M_{r}(F)$. In particular, $(A, \sigma)$ contains $(Q,-)$.

Focusing on the anisotropic case in the propositions above, we have:

Corollary 2.3. Let $(A, \sigma)$ be a central simple F-algebra with involution. Assume $\sigma$ is anisotropic and $(A, \sigma)_{F_{Q}}$ is split hyperbolic. Then either

- $A$ is split, $\sigma$ is orthogonal, and there is a quadratic form $q$ such that

$$
(A, \sigma) \simeq \operatorname{Ad}_{n_{Q} \otimes q} \simeq(Q,-) \otimes(Q,-) \otimes \operatorname{Ad}_{q} ;
$$

or

- A is Brauer-equivalent to $Q, \sigma$ is symplectic, and there is a quadratic form $q$ such that

In both cases, $(A, \sigma)$ contains $(Q,-)$.

$$
(A, \sigma) \simeq(Q,-) \otimes \operatorname{Ad}_{q} .
$$

Using the results in this section, we may describe the algebras with involution of degree $2 \bmod 4$ that become hyperbolic over $F_{Q}$ :

Corollary 2.4. Let $(A, \sigma)$ be a central simple $F$-algebra with involution. If $\operatorname{deg} A \equiv$ $2 \bmod 4$ and $(A, \sigma)_{F_{Q}}$ is hyperbolic, then $A_{F_{Q}}$ is split. If moreover $\sigma$ is anisotropic, then it is symplectic and there is an odd-dimensional quadratic form $q$ over $F$ such that $(A, \sigma) \simeq(Q,-) \otimes \operatorname{Ad}_{q}$. 
Proof. Since $\operatorname{deg} A \equiv 2 \bmod 4$, we have $A \simeq M_{r}(H)$ for some odd integer $r$ and some quaternion algebra $H$ over $F$. If $H$ is division, $A$ does not admit a hyperbolic involution. Therefore, the hypothesis that $(A, \sigma)_{F_{Q}}$ is hyperbolic implies that $H_{F_{Q}}$ is split, hence $(A, \sigma)_{F_{Q}}$ is split hyperbolic. The last statement then readily follows from Corollary 2.3.

In view of this corollary, we only consider central simple algebras of degree divisible by 4 in the following sections.

\section{Algebras of Degree 4}

Throughout this section, $A$ is a central simple algebra of degree 4 over an arbitrary field $F$ of characteristic different from 2. Involutions on $A$ are classified by cohomological invariants (see [11, Ch. 4]), which we use to give an explicit description of the involutions on $A$ that become hyperbolic over $F_{Q}$.

3.1. The orthogonal case. This case is easy to handle using Clifford algebras.

Proposition 3.1. Let $\sigma$ be an orthogonal involution on $A$.

(1) $(A, \sigma)$ is hyperbolic if and only if $\operatorname{disc} \sigma=1$ and one of the components of $C(A, \sigma)$ is split;

(2) $(A, \sigma)$ contains $(Q,-)$ if and only if $\operatorname{disc} \sigma=1$ and one of the components of $C(A, \sigma)$ is isomorphic to $Q$;

(3) A contains $Q$ if and only if $A \otimes_{F} Q$ is Brauer-equivalent to a quaternion algebra.

Proof. (1) This readily follows from [2, 2.5].

(2) If $(A, \sigma)$ contains $(Q,-)$, then there is a quaternion $F$-algebra $Q^{\prime}$ with canonical involution - such that

$$
(A, \sigma) \simeq(Q,-) \otimes\left(Q^{\prime},-\right)
$$

By [11, (15.12)], this relation holds if and only if $C(A, \sigma) \simeq Q \times Q^{\prime}$. This proves (2).

(3) If $A$ contains (a copy of) $Q$, then the centralizer of $Q$ in $A$ is a quaternion $F$-algebra $Q^{\prime}$, and we have

$$
A \simeq Q \otimes_{F} Q^{\prime}
$$

This relation holds if and only if $A \otimes_{F} Q$ is Brauer-equivalent to $Q^{\prime}$, hence (3) follows.

Corollary 3.2. Let $\sigma$ be an orthogonal involution on $A$. Then $(A, \sigma)_{F_{Q}}$ is hyperbolic if and only if $(A, \sigma)$ is hyperbolic or contains $(Q,-)$.

Proof. As noticed in the introduction, any $(A, \sigma)$ containing $(Q,-)$ is hyperbolic over $F_{Q}$, so we only have to prove the converse. Assume $(A, \sigma)_{F_{Q}}$ hyperbolic. Since $F$ is quadratically closed in $F_{Q}$, the discriminant of $\sigma$ is trivial, so $C(A, \sigma)$ is a direct product of two quaternion algebras. At least one of these quaternion algebras splits over $F_{Q}$ by Proposition 3.1(1), hence that component must be either split or isomorphic to $Q$. It follows that $(A, \sigma)$ is either hyperbolic or contains $(Q,-)$, by Proposition 3.1.

Note that a central simple algebra of degree 4 with hyperbolic involution does not necessarily contain $(Q,-)$, even if it contains $Q$ : if $Q^{\prime}$ is a quaternion $F$-algebra such that $Q \otimes{ }_{F} Q^{\prime}$ has index 2 , then $(A, \sigma)=\left(M_{2}(F),-\right) \otimes\left(Q^{\prime},-\right)$ is hyperbolic over $F$ and satisfies $C(A, \sigma) \simeq M_{2}(F) \times Q^{\prime}$ by [11, (15.12)]. Therefore, Proposition 3.1] (2) 
shows that $(A, \sigma)$ does not contain $(Q,-)$, even though Proposition 3.1(3) shows that $A$ contains $Q$. This situation does not occur in the symplectic case, in view of Proposition 3.3 below.

3.2. The symplectic case. Symplectic involutions on $A$ are classified up to conjugation by a relative invariant $\Delta$ with values in the Galois cohomology group $H^{3}\left(F, \mu_{2}\right)$, see ${ }^{1}$ [11, (16.9)]. We denote by $[A]$ the Brauer class of $A$, viewed as an element of $H^{2}\left(F, \mu_{2}\right)$, and for $\lambda \in F^{\times}$we denote by $(\lambda)$ the square class of $\lambda$, viewed as an element in $H^{1}\left(F, \mu_{2}\right)$. Using the invariant $\Delta$, we show:

Proposition 3.3. Let $\sigma$ be a symplectic involution on $A$. If $A$ contains $Q$ and $(A, \sigma)_{F_{Q}}$ is hyperbolic, then $(A, \sigma)$ contains $(Q,-)$.

Proof. Since $A$ contains $Q$, it decomposes as $A=Q \otimes Q^{\prime}$ for some quaternion algebra $Q^{\prime}=\left(a^{\prime}, b^{\prime}\right)_{F}$. Let $\tau^{\prime}$ be an orthogonal involution on $Q^{\prime}$ of discriminant $a^{\prime}$. The involution $\tau=-\otimes \tau^{\prime}$ on $A=Q \otimes Q^{\prime}$ is of symplectic type, and clearly hyperbolic over $F_{Q}$ since $(A, \tau)$ contains $(Q,-)$. Similarly, for every invertible $y \in \operatorname{Sym}\left(Q^{\prime}, \tau^{\prime}\right)$, the involution

$$
\tau_{y}=\operatorname{Int}(1 \otimes y) \circ \tau=-\otimes(\operatorname{Int}(y) \circ \tau)
$$

is such that $\left(A, \tau_{y}\right)$ contains $(Q,-)$. We prove below that $\sigma$ is conjugate to $\tau_{y}$ for a suitable $y$. By [11, (16.18)], the relative discriminant $\Delta_{\tau}\left(\tau^{\prime}\right)$ is given by

$$
\Delta_{\tau}\left(\tau^{\prime}\right)=\left(\operatorname{Nrp}_{\tau}(1 \otimes y)\right) \cup[A] \in H^{3}\left(F, \mu_{2}\right),
$$

where $\mathrm{Nrp}_{\tau}$ is the Pfaffian norm, as defined in [11, (2.9)]. Since this relative discriminant classifies symplectic involutions on $A$ up to conjugation, it suffices to show:

Lemma 3.4. There exists an invertible element $y \in \operatorname{Sym}\left(Q^{\prime}, \tau^{\prime}\right)$ such that

$$
\Delta_{\tau}(\sigma)=\left(\operatorname{Nrp}_{\tau}(1 \otimes y)\right) \cup[A] .
$$

Proof. The involution $\sigma$ can be written as $\sigma=\operatorname{Int}(x) \circ \tau$ for some $x \in \operatorname{Sym}(A, \sigma)$, hence we already have

$$
\Delta_{\tau}(\sigma)=\left(\operatorname{Nrp}_{\tau}(x)\right) \cup[A],
$$

and we want to prove we can substitute for $x$ some element $1 \otimes y$ with $y \in$ $\operatorname{Sym}\left(Q^{\prime}, \tau^{\prime}\right)$. Since both $\sigma$ and $\tau$ become hyperbolic over $F_{Q}$, the relative discriminant $\Delta_{\tau}(\sigma)$ is killed by $F_{Q}$, hence $\left(\operatorname{Nrp}_{\tau}(x)\right) \cup\left[Q^{\prime}\right]=(\lambda) \cup[Q]$ for some $\lambda \in F^{\times}$. By the common slot lemma [1, Lemma 1.7], we may even assume

$$
\left(\operatorname{Nrp}_{\tau}(x)\right) \cup\left[Q^{\prime}\right]=(\lambda) \cup\left[Q^{\prime}\right]=(\lambda) \cup[Q],
$$

from which we deduce

$$
(\lambda) \cup\left([Q]+\left[Q^{\prime}\right]\right)=0, \quad \text { and } \quad\left(\lambda \operatorname{Nrp}_{\tau}(x)\right) \cup\left[Q^{\prime}\right]=0 .
$$

Hence the relative discriminant of $\sigma$ is

$\Delta_{\tau}(\sigma)=\left(\operatorname{Nrp}_{\tau}(x)\right) \cup\left([Q]+\left[Q^{\prime}\right]\right)=\left(\lambda \operatorname{Nrp}_{\tau}(x)\right) \cup\left([Q]+\left[Q^{\prime}\right]\right)=\left(\lambda \operatorname{Nrp}_{\tau}(x)\right) \cup[Q]$.

Moreover, the quadratic space $\left(\operatorname{Sym}(A, \sigma), \operatorname{Nrp}_{\tau}\right)$ is an Albert quadratic space for the biquaternion algebra $A$ by [1], (16.8)]. Hence, its Clifford invariant is $e_{2}\left(\operatorname{Nrp}_{\tau}\right)=[A]=[Q]+\left[Q^{\prime}\right]$. We deduce from (5) that the quadratic form $\langle\langle\lambda\rangle\rangle \otimes \operatorname{Nrp}_{\tau}$ has trivial Arason invariant $e_{3}$, and hence is hyperbolic by the Arason-Pfister

\footnotetext{
${ }^{1}$ The discussion in [1] is in terms of the 3 -fold Pfister form $j$ whose Arason invariant is $\Delta$.
} 
Hauptsatz. So there exists an element $x^{\prime} \in \operatorname{Sym}(A, \sigma)$ such that $\lambda \operatorname{Nrp}_{\tau}(x)=$ $\operatorname{Nrp}_{\tau}\left(x^{\prime}\right)$, and we have proven

$$
\Delta_{\tau}(\sigma)=\left(\operatorname{Nrp}_{\tau}\left(x^{\prime}\right)\right) \cup[Q] \text { and }\left(\operatorname{Nrp}_{\tau}\left(x^{\prime}\right)\right) \cup\left[Q^{\prime}\right]=0
$$

for some $x^{\prime} \in \operatorname{Sym}(A, \sigma)$.

Let us now pick a pure quaternion $i^{\prime} \in Q^{\prime}$ such that $i^{\prime 2}=a^{\prime}$ and $\tau^{\prime}=\operatorname{Int}\left(i^{\prime}\right) \circ-$. Denoting by $Q^{0}$ the vector space of pure quaternions in $Q$, we have

$$
\operatorname{Sym}(A, \tau)=\left(1 \otimes \operatorname{Sym}\left(Q^{\prime}, \tau^{\prime}\right)\right) \oplus\left(Q^{0} \otimes i^{\prime}\right) .
$$

Hence $x^{\prime}$ can be written as $x^{\prime}=x_{0}+1 \otimes \xi_{1}+\xi_{2} \otimes i^{\prime}$ for some $x_{0} \in F$, some pure quaternion $\xi_{1} \in Q^{\prime 0} \cap \operatorname{Sym}\left(Q^{\prime}, \tau^{\prime}\right)$ and some $\xi_{2} \in Q^{0}$. The pure quaternion $\xi_{1}$ being $\tau^{\prime}$-symmetric, it anticommutes with $i^{\prime}$, and changing of quaternionic basis if necessary, we may assume that $\xi_{1}^{2}=b^{\prime} x_{1}^{2}$ for some $x_{1} \in F$. Similarly, we may assume that $\xi_{2}^{2}=a x_{2}^{2}$ for some $x_{2} \in F$. Note that we allow $x_{i}=0$ for $i=0,1$, and 2 since some term might be zero in the decomposition of $x^{\prime}$. We then have

$$
\operatorname{Nrp}_{\tau}\left(x^{\prime}\right)=x_{0}^{2}-b^{\prime} x_{1}^{2}-a a^{\prime} x_{2}^{2},
$$

and the following lemma finishes the proof:

Lemma 3.5. There exists $z_{0}, z_{1}, y_{0}, y_{1}$ and $y_{2}$ such that $\operatorname{Nrp}_{\tau}\left(x^{\prime}\right)\left(z_{0}^{2}-a z_{1}^{2}\right)=$ $y_{0}^{2}-b^{\prime} y_{1}^{2}+a^{\prime} b^{\prime} y_{2}^{2} \neq 0$.

Indeed, if we let $y=y_{0}+y_{1} j^{\prime}+y_{2} i^{\prime} j^{\prime}$, where $j^{\prime}$ is a pure quaternion in $Q^{\prime}$ such that $j^{\prime 2}=b^{\prime}$ and $i^{\prime} j^{\prime}=-j^{\prime} i^{\prime}$, we have $\operatorname{Nrp}_{\tau}(1 \otimes y)=N r d_{Q^{\prime}}(y)=y_{0}^{2}-b^{\prime} y_{1}^{2}+a^{\prime} b^{\prime} y_{2}^{2}$ (see [1], (2.11)]). Hence,

$$
\begin{aligned}
\Delta_{\tau}(\sigma) & =\left(\operatorname{Nrp}_{\tau}\left(x^{\prime}\right)\right) \cup\left[(a, b)_{F}\right]=\left(\operatorname{Nrp}_{\tau}\left(x^{\prime}\right)\left(z_{0}^{2}-a z_{1}^{2}\right)\right) \cup\left[(a, b)_{F}\right] \\
& =\left(\operatorname{Nrd}_{Q^{\prime}}(y)\right) \cup[A]=\left(\operatorname{Nrp}_{\tau}(1 \otimes y)\right) \cup[A], \quad \text { with } y \in \operatorname{Sym}\left(Q^{\prime}, \tau^{\prime}\right) .
\end{aligned}
$$

Proof of Lemma 3. . If $\left\langle 1,-b^{\prime}, a^{\prime} b^{\prime}\right\rangle$ is isotropic, then it is universal, hence represents $\operatorname{Nrp}_{\tau}\left(x^{\prime}\right)$ and we are done. Otherwise, denote $\mu=\operatorname{Nrp}_{\tau}\left(x^{\prime}\right)$; by (7), we have $(\mu) \cup\left[\left(a a^{\prime}, b^{\prime}\right)_{F}\right]=0$, and combining with (6), we get $(\mu) \cup\left[\left(a, b^{\prime}\right)\right]=0$. In terms of quadratic forms, this means that the 3 -fold Pfister form

$$
\left\langle\left\langle\mu, a, b^{\prime}\right\rangle\right\rangle=\left\langle 1,-\mu,-a, \mu b^{\prime}\right\rangle \perp\left\langle\mu a, a b^{\prime},-\mu a b^{\prime}\right\rangle \perp\left\langle-b^{\prime}\right\rangle
$$

is hyperbolic. On the other hand, by (雨), the quadratic form $\left\langle 1,-b^{\prime},-a a^{\prime},-\mu\right\rangle$ is isotropic. Multiplying by $\left(-a b^{\prime} \mu\right)$, we get that $-a^{\prime} b^{\prime} \mu$ is represented by the form $\left\langle\mu a, a b^{\prime},-\mu a b^{\prime}\right\rangle$. Hence, in view of (8), the quadratic form $\left\langle 1,-\mu,-a, \mu b^{\prime},-a^{\prime} b^{\prime} \mu\right\rangle$, which is a 5 -dimensional subform of $\left\langle\left\langle\mu, a, b^{\prime}\right\rangle\right\rangle$, is necessarily isotropic. Since the quadratic forms $\langle 1,-a\rangle$ and $\left\langle 1,-b^{\prime}, a^{\prime} b^{\prime}\right\rangle$ are anisotropic, this completes the proof.

Using Proposition 3.3, we may characterize the symplectic involutions on $A$ that become hyperbolic over $F_{Q}$ :

Theorem 3.6. Let $\sigma$ be a symplectic involution on A. If $(A, \sigma)_{F_{Q}}$ is hyperbolic, then either

(a) $(A, \sigma) \simeq(Q,-) \otimes\left(Q^{\prime}, \rho\right)$ for some quaternion F-algebra with orthogonal involution $\left(Q^{\prime}, \rho\right)$, or

(b) $(A, \sigma) \simeq \operatorname{Ad}_{\langle\langle\lambda\rangle\rangle} \otimes\left(Q^{\prime},-\right)$ for some quaternion $F$-algebra $Q^{\prime}$ and some $\lambda \in$ $F^{\times}$with the following properties: $Q \otimes_{F} Q^{\prime}$ is a division algebra and the norm forms $n_{Q}, n_{Q^{\prime}}$ satisfy $\langle\langle\lambda\rangle\rangle \cdot n_{Q} \simeq\langle\langle\lambda\rangle\rangle \cdot n_{Q^{\prime}}$. 
Conversely, if $(A, \sigma)$ is of either type above, then $(A, \sigma)_{F_{Q}}$ is hyperbolic.

Note that (a) and (b) are mutually exclusive since in the first case $A \otimes_{F} Q$ has Schur index 1 or 2, whereas it has index 4 in case (b).

Proof. Suppose $(A, \sigma)$ is not as in case (a) and $(A, \sigma)_{F_{Q}}$ is hyperbolic. Then Proposition 3.3 shows that $A$ does not contain $Q$. In particular, $A$ is not division, by Proposition 1.2, so $A \simeq M_{2}\left(Q^{\prime}\right)$ for some quaternion $F$-algebra $Q^{\prime}$, and $Q \otimes_{F} Q^{\prime}$ is a division algebra by Proposition 3.1 (3). As observed in the proof of Proposition $2.1(2),(A, \sigma)$ contains $\left(Q^{\prime},-\right)$, hence

$$
(A, \sigma) \simeq \operatorname{Ad}_{\langle\langle\lambda\rangle\rangle} \otimes\left(Q^{\prime},-\right) \quad \text { for some } \lambda \in F^{\times} .
$$

The algebra $A$ carries a hyperbolic symplectic involution $\tau$, and we have by [1]. $(16.21)]$

$$
\Delta_{\tau}(\sigma)=(\lambda) \cup\left[Q^{\prime}\right] .
$$

Since $(A, \sigma)_{F_{Q}}$ is hyperbolic, this invariant vanishes over $F_{Q}$. Hence, by 12 . $\mathrm{X}(4.11)]$ and Arason's common slot lemma we may assume as in the proof of Lemma 3.4 that $(\lambda) \cup\left[Q^{\prime}\right]=(\lambda) \cup[Q]$. Therefore, $\langle\langle\lambda\rangle\rangle \cdot n_{Q^{\prime}} \simeq\langle\langle\lambda\rangle\rangle \cdot n_{Q}$, which shows that $(A, \sigma)$ is as in case (b).

It is clear that $(A, \sigma)_{F_{Q}}$ is hyperbolic in case (a). In case (b) the algebra $A$ carries a hyperbolic symplectic involution $\tau$ and $\Delta_{\tau}(\sigma)=(\lambda) \cup\left[Q^{\prime}\right]$ vanishes over $F_{Q}$. Therefore, $(A, \sigma)_{F_{Q}}$ is hyperbolic.

\section{4. $F_{Q}$-Minimal QUADRATIC Forms of Dimension 5}

A quadratic form $\varphi$ over $F$ is called $F_{Q}$-minimal if $\varphi_{F_{Q}}$ is isotropic and $\psi_{F_{Q}}$ is anisotropic for every proper subform $\psi \subset \varphi$. In this section, we show that Theorem 3.6 can be used to recover (and is in fact equivalent to) the description of $F_{Q}$-minimal forms of dimension 5 due to Hoffmann, Lewis, and Van Geel [9. Prop. 4.1].

A general procedure to construct central simple algebras with involution that become hyperbolic over $F_{Q}$ uses Clifford algebras. Recall from [12, V(1.9), V(2.4)] that for any quadratic form $\varphi$ of odd dimension $2 m+1$ over $F$ the even Clifford algebra $C_{0}(\varphi)$ is central simple over $F$ of degree $2^{m}$. It carries a canonical involution $\tau_{0}$, which is the restriction of the involution on the full Clifford algebra that leaves invariant every vector in the underlying vector space of $\varphi$. The involution $\tau_{0}$ is orthogonal if $m \equiv 0$ or $3 \bmod 4$ and symplectic otherwise, see [11] (8.4)].

Proposition 4.1. Let $\varphi$ be a quadratic form of odd dimension over $F$.

(1) If $\varphi_{F_{Q}}$ is isotropic, then $\left(C_{0}(\varphi), \tau_{0}\right)_{F_{Q}}$ is hyperbolic. The converse holds if $\operatorname{dim} \varphi=5$.

(2) If $\varphi$ contains a subform similar to $\langle 1,-a,-b\rangle$, then $\left(C_{0}(\varphi), \tau_{0}\right)$ contains $(Q,-)$. The converse holds if $\operatorname{dim} \varphi=5$.

Proof. (1) The first statement readily follows from [11, (8.5)] and the second from 11. (15.21)].

(2) Suppose the underlying vector space of $\varphi$ contains orthogonal vectors $e_{0}, e_{1}$, $e_{2}$ satisfying for some $\lambda \in F^{\times}$

$$
\varphi\left(e_{0}\right)=\lambda, \quad \varphi\left(e_{1}\right)=-\lambda a, \quad \varphi\left(e_{2}\right)=-\lambda b .
$$

Then the products $e_{0} e_{1}$ and $e_{0} e_{2}$ generate a $\tau_{0}$-stable subalgebra of $C_{0}(\varphi)$ isomorphic to $(Q,-)$. 
For the rest of the proof, suppose $\operatorname{dim} \varphi=5$ and $\left(C_{0}(\varphi), \tau_{0}\right)$ contains $(Q,-)$. The centralizer of $(Q,-)$ is a quaternion algebra with orthogonal involution $\left(Q^{\prime}, \rho\right)$ such that

$$
\left(C_{0}(\varphi), \tau_{0}\right)=(Q,-) \otimes\left(Q^{\prime}, \rho\right)
$$

Let $V \subset \operatorname{Sym}\left(C_{0}(\varphi), \tau_{0}\right)$ be the vector space of $\tau_{0}$-symmetric elements of trace 0 . The map $x \mapsto x^{2}$ defines a quadratic form $s: V \rightarrow F$ that is similar to $\varphi$ by the equivalence $\mathrm{B}_{2} \equiv \mathrm{C}_{2}$, see [11, (15.16)]. Let $y \in Q^{\prime}$ be a $\rho$-skew-symmetric unit and let $Q^{0} \subset Q$ be the vector space of pure quaternions. The restriction of $s$ to the subspace $Q^{0} \otimes y \subset V$ is similar to $\langle 1,-a,-b\rangle$, hence the proof is complete.

Corollary 4.2 (Hoffmann-Lewis-Van Geel [9, Prop. 4.1]). A 5-dimensional quadratic form $\varphi$ over $F$ is $F_{Q}$-minimal if and only if the following conditions hold:

(a) $\varphi$ is similar to a Pfister neighbour of the 3 -fold Pfister form $\langle\langle a, b, \lambda\rangle$ for some $\lambda \in F^{\times}$, and

(b) $C_{0}(\varphi) \simeq M_{2}\left(Q^{\prime}\right)$ for some quaternion $F$-algebra $Q^{\prime}$ such that $Q \otimes_{F} Q^{\prime}$ is a division algebra.

Proof. Proposition 4.1 shows that $\varphi$ is $F_{Q}$-minimal if and only if $\left(C_{0}(\varphi), \tau_{0}\right)$ is hyperbolic but $\left(C_{0}(\varphi), \tau_{0}\right)$ does not contain $(Q,-)$. By Theorem 3.6 , this condition is equivalent to

$$
\left(C_{0}(\varphi), \tau_{0}\right) \simeq \operatorname{Ad}_{\langle\lambda\rangle\rangle} \otimes\left(Q^{\prime},-\right)
$$

for some quaternion $F$-algebra $Q^{\prime}=\left(a^{\prime}, b^{\prime}\right)_{F}$ and some $\lambda \in F^{\times}$with $Q \otimes Q^{\prime}$ a division algebra and $\langle\langle a, b, \lambda\rangle\rangle \simeq\left\langle\left\langle a^{\prime}, b^{\prime}, \lambda\right\rangle\right\rangle$. It follows from the isomorphism (9) that $\varphi$ is similar to a Pfister neighbour of $\left\langle\left\langle a^{\prime}, b^{\prime}, \lambda\right\rangle\right\rangle$, by [11, p. 271]. Thus, (a) and (b) hold if $\varphi$ is $F_{Q^{-m i n i m a l}}$. Conversely, if $C_{0}(\varphi) \simeq M_{2}\left(Q^{\prime}\right)$ for some quaternion $F$-algebra $Q^{\prime}=\left(a^{\prime}, b^{\prime}\right)_{F}$, then as observed in the proof of Proposition 2.1. (2) we have

$$
\left(C_{0}(\varphi), \tau_{0}\right) \simeq \operatorname{Ad}_{\langle\langle\mu\rangle\rangle} \otimes\left(Q^{\prime},-\right) \quad \text { for some } \mu \in F^{\times} .
$$

It then follows from [11, p. 271] that $\varphi$ is similar to a Pfister neighbour of $\left\langle\left\langle a^{\prime}, b^{\prime}, \mu\right\rangle\right\rangle$. If (a) holds, then $\langle\langle a, b, \lambda\rangle\rangle\left\langle\left\langle a^{\prime}, b^{\prime}, \mu\right\rangle\right\rangle$ and by the common slot lemma we may assume $\lambda=\mu$. Thus, $\varphi$ is $F_{Q}$-minimal if (a) and (b) hold.

\section{Totally Decomposable orthogonal involutions of Degree 8}

In this section, $A$ denotes a central simple $F$-algebra of degree 8 and $\sigma$ is an orthogonal involution on $A$. The algebra with involution $(A, \sigma)$ is called totally decomposable if there are $\sigma$-stable quaternion subalgebras $Q_{1}, Q_{2}, Q_{3}$ in $A$ such that $A=Q_{1} \otimes Q_{2} \otimes Q_{3}$. Denoting by $\sigma_{i}$ the restriction of $\sigma$ to $Q_{i}$ for $i=1,2,3$, we then have

$$
(A, \sigma)=\left(Q_{1}, \sigma_{1}\right) \otimes\left(Q_{2}, \sigma_{2}\right) \otimes\left(Q_{3}, \sigma_{3}\right)
$$

The totally decomposable algebras of degree 8 are characterized by the property that $\operatorname{disc} \sigma=1$ and one of the components of the Clifford algebra $C(A, \sigma)$ is split, see [11, (42.11)]. Proposition 5.1 below shows how to use this criterion to relate totally decomposable algebras to quadratic forms.

Recall that for any quadratic form $\varphi$ of dimension 8 with $\operatorname{disc} \varphi=1$ the even Clifford algebra decomposes into a direct product of central simple $F$-algebras of degree 8,

$$
C_{0}(\varphi) \simeq C_{+}(\varphi) \times C_{-}(\varphi)
$$


The canonical involution $\tau_{0}$ on $C_{0}(\varphi)$ restricts to orthogonal involutions $\tau_{+}, \tau_{-}$on $C_{+}(\varphi)$ and $C_{-}(\varphi)$, and we have

$$
\left(C_{+}(\varphi), \tau_{+}\right) \simeq\left(C_{-}(\varphi), \tau_{-}\right) .
$$

It is easily checked that $\left(C_{+}(\varphi), \tau_{+}\right)$is totally decomposable.

Proposition 5.1. For every central simple algebra of degree 8 with totally decomposable orthogonal involution $(A, \sigma)$, there is a quadratic form $\varphi$ with $\operatorname{dim} \varphi=8$ and $\operatorname{disc} \sigma=1$ such that

$$
(A, \sigma) \simeq\left(C_{+}(\varphi), \tau_{+}\right)
$$

The form $\varphi$ is uniquely determined by $(A, \sigma)$ up to similarity. Moreover,

- the algebra $A$ is split if and only if $\varphi$ is a multiple of a 3-fold Pfister form; in that case $(A, \sigma) \simeq \operatorname{Ad}_{\varphi}$;

- the algebra $(A, \sigma)$ contains $(Q,-)$ if and only if $\varphi$ contains a subform similar to $\langle 1,-a,-b\rangle$.

Furthermore, the following conditions are equivalent:
(a) $(A, \sigma)$ is isotropic;
(b) $(A, \sigma)$ is hyperbolic;
(c) $\varphi$ is isotropic.

Proof. Since $(A, \sigma)$ is totally decomposable, it follows from [11, (42.11)] that one of the components $C_{+}(A, \sigma)$ of the Clifford algebra is split. The canonical involution $\sigma_{+}$on $C_{+}(A, \sigma)$ is orthogonal, hence there is a quadratic form $\varphi$ of dimension 8 such that

By triality (see [1], (42.3)]) we have

$$
\left(C_{+}(A, \sigma), \sigma_{+}\right) \simeq \operatorname{Ad}_{\varphi} .
$$

$$
\left(C_{0}(\varphi), \tau_{0}\right) \simeq(A, \sigma) \times(A, \sigma) .
$$

Therefore, $\operatorname{disc} \varphi=1$ and $(A, \sigma) \simeq\left(C_{+}(\varphi), \tau_{+}\right)$. Conversely, triality also shows that if $(A, \sigma) \simeq\left(C_{+}(\varphi), \tau_{+}\right)$, then the canonical involution $\underline{\sigma}$ on $C(A, \sigma)$ satisfies

$$
(C(A, \sigma), \underline{\sigma}) \simeq \operatorname{Ad}_{\varphi} \times(A, \sigma),
$$

hence the form $\varphi$ is uniquely determined up to similarity.

The Clifford algebra of $\varphi$ splits if and only if $\varphi$ is a multiple of a 3 -fold Pfister form, by [16, Ch. 2, Th. 14.4] and [12, X(5.6)]. When that condition holds we have $(A, \sigma) \simeq \widehat{\operatorname{Ad}}_{\varphi}$ by [11, (35.1)].

If $\varphi$ contains a multiple of $\langle 1,-a,-b\rangle$, then the same argument as in the proof of Proposition 4.1 (2) shows that $\left(C_{0}(\varphi), \tau_{0}\right)$ contains $(Q,-)$. Projecting on each component, it follows that $\left(C_{+}(\varphi), \tau_{+}\right)$contains $(Q,-)$. Conversely, if $(A, \sigma)$ contains $(Q,-)$, then we have

$$
(A, \sigma)=(Q,-) \otimes\left(A_{1}, \sigma_{1}\right)
$$

for some central simple algebra with symplectic involution $\left(A_{1}, \sigma_{1}\right)$ of degree 4 . By [1. (15.19)] there is a 5-dimensional quadratic form $\psi$ such that disc $\psi=1$ and $\left(A_{1}, \sigma_{1}\right) \simeq\left(C_{0}(\psi), \tau_{0}\right)$. Letting $\tau_{0}^{\prime}$ be the canonical involution on $C_{0}(\langle a b,-a,-b\rangle)$, we may rewrite (10) as

$$
(A, \sigma) \simeq\left(C_{0}(\langle a b,-a,-b\rangle), \tau_{0}^{\prime}\right) \otimes\left(C_{0}(\psi), \tau_{0}\right) .
$$

In view of the canonical embedding

$$
C_{0}(\langle a b,-a,-b\rangle) \otimes_{F} C_{0}(\psi) \hookrightarrow C_{0}(\langle a b,-a,-b\rangle \perp \psi),
$$


which is compatible with the canonical involutions, (11) yields

$$
(A, \sigma) \simeq\left(C_{+}(\langle a b,-a,-b\rangle \perp \psi), \tau_{+}\right) .
$$

Uniqueness of $\varphi$ shows that $\varphi$ is similar to $\langle a b,-a,-b\rangle \perp \psi$, hence it contains a subform similar to $\langle 1,-a,-b\rangle$.

The equivalence of (a), (b), (c) is clear if $A$ is split, since then $(A, \sigma) \simeq \operatorname{Ad}_{\varphi}$ and $\varphi$ is a 3 -fold Pfister form, hence it is isotropic if and only if it is hyperbolic. For the rest of the proof, we may thus assume $A$ is not split. If $(A, \sigma)$ is hyperbolic, then it follows from [ [6] that the split component of $(C(A, \sigma), \underline{\sigma})$ is isotropic, hence (b) $\Rightarrow(\mathrm{c})$. Conversely, if (c) holds, then [11, (8.5)] shows that $\left(C_{0}(\varphi), \tau_{0}\right)$ is hyperbolic, hence $\left(C_{+}(\varphi), \tau_{+}\right)$also is hyperbolic, proving $(\mathrm{c}) \Rightarrow(\mathrm{b})$. The equivalence of (a) and (b) readily follows from [3, Prop. 2.10].

To give an example of a division $F$-algebra of degree 8 with a totally decomposable orthogonal involution that is hyperbolic over $F_{Q}$ but does not contain $(Q,-)$, we use an example of $F_{Q}$-minimal quadratic form of dimension 7 due to Hoffmann and Van Geel [10]. For the rest of this section, we fix the following notation: $F_{1}=F_{0}(t, u)$ is the function field in two independent indeterminates over an arbitrary field $F_{0}$ of characteristic different from 2 , and $F=F_{1}((a))((b))$ is the iterated Laurent series field in two indeterminates $a, b$. In accordance with our running notation, $Q$ denotes the quaternion algebra $(a, b)_{F}$. Let

$$
\varphi_{0}=\langle 1+t, u\rangle \perp\langle-a\rangle\langle 1, u\rangle \perp\langle-b\rangle\langle 1, t+u\rangle \perp\langle a b\rangle\langle t\rangle
$$

(see [10, p. 43]) and

$$
\varphi=\varphi_{0} \perp\langle a b\rangle\langle t(1+t)(t+u)\rangle,
$$

so $\operatorname{dim} \varphi=8$ and $\operatorname{disc} \varphi=1$. Let also

$$
(A, \sigma)=\left(C_{+}(\varphi), \tau_{+}\right) \quad\left(=\left(C_{0}\left(\varphi_{0}\right), \tau_{0}\right)\right)
$$

a central simple $F$-algebra of degree 8 with a totally decomposable involution.

Theorem 5.2. The algebra with involution $(A, \sigma)$ does not contain $(Q,-)$, yet $(A, \sigma)_{F_{Q}}$ is hyperbolic. Moreover, $A$ is a division algebra.

Proof. Since $\langle 1, t\rangle \simeq\langle 1+t, t(1+t)\rangle$ and $\langle t, u\rangle \simeq\langle t+u, t u(t+u)\rangle$, we have

$$
\varphi_{0} \perp\langle t(1+t),-a t,-b t u(t+u), a b, a b u\rangle \simeq\langle 1, t, u\rangle\langle\langle a, b\rangle\rangle .
$$

Since the right side is hyperbolic over $F_{Q}$, it follows that $\left(\varphi_{0}\right)_{F_{Q}}$ is isotropic, and therefore $(A, \sigma)_{F_{Q}}$ is hyperbolic by Proposition 4.1(1) or 5.1.

To show $(A, \sigma)$ does not contain $(Q,-)$, we prove $\varphi$ does not contain any subform similar to $\langle 1,-a,-b\rangle$. As in [10, p. 43], we decompose $\varphi$ as

$$
\varphi=\alpha \perp\langle-a\rangle \beta \perp\langle-b\rangle \gamma \perp\langle a b\rangle \delta,
$$

where $\alpha=\langle 1+t, u\rangle, \beta=\langle 1, u\rangle, \gamma=\langle 1, t+u\rangle$, and $\delta=\langle t, t(1+t)(t+u)\rangle$. By Springer's theorem, the isometry classes of $\alpha, \beta, \gamma$, and $\delta$ over $F_{1}$ are uniquely determined by $\varphi$. If three of those quadratic forms represent a common value $\lambda$, then $\varphi$ contains a 3 -dimensional subform of $\langle\lambda\rangle\langle\langle a, b\rangle\rangle$, hence a subform similar to $\langle 1,-a,-b\rangle$. We claim that the converse also holds. Indeed, assume first that $\varphi$ contains $\langle\lambda\rangle\langle 1,-a,-b\rangle$ for some $\lambda \in F_{1}^{\times}$. Writing $\varphi=\langle\lambda\rangle\langle 1,-a,-b\rangle \perp \varphi^{\prime}$ as in (13), we get, by uniqueness of the forms $\alpha, \beta$ and $\gamma$ up to isometry, that all three represent $\lambda$. Consider now the general situation, where $\lambda \in F^{\times}$need not be in $F_{1}$; 
modifying it by a square if necessary, we may write it as $\lambda_{0},-a \lambda_{0},-b \lambda_{0}$ or $a b \lambda_{0}$ for some $\lambda_{0} \in F_{1}^{\times}$. The same argument as above then proves the claim.

Thus, to prove that $\varphi$ does not contain any subform similar to $\langle 1,-a,-b\rangle$, we have to show that no three of the quadratic forms $\alpha, \beta, \gamma$, and $\delta$ have any common value over $F_{1}$. This can be checked after some scalar extension. For instance, by 10 , Lemma(4.4)(iii)], the only common value of $\alpha$ and $\beta$ over $F_{0}(t)((u))$ is the square class of $u$. On the other hand, applying [12, $\mathrm{VI}(1.3)]$, one may check that $\gamma$ and $\delta$ are both isomorphic over $F_{0}((t))((u))$ to $\langle 1, t\rangle$, which does not represent $u$. On the other hand, over $F_{0}(t)((t+u))$ the form $\gamma$ only represents the square classes of 1 and $t+u$, whereas $\delta$ only represents the square classes of $t$ and $t(1+t)(t+u)$. Therefore, $\gamma$ and $\delta$ have no common value in $F_{1}$.

To complete the proof, we show $A$ is a division algebra. Taking the Clifford invariant of each side of (12) and applying [12, $\mathrm{V}(3.13)]$, we obtain the following equality in the Brauer group of $F$ :

$$
[A]+\left[C_{0}(\langle t(1+t),-a t,-b t u(t+u), a b, a b u\rangle)\right]=[Q] .
$$

The even Clifford algebra of the 5-dimensional form is easily computed:

$$
C_{0}(\langle t(1+t),-a t,-b t u(t+u), a b, a b u\rangle) \simeq(-u, b t)_{F} \otimes(a b(t+u),-a u(1+t))_{F},
$$

hence

$$
\begin{aligned}
A & \simeq(a, b)_{F} \otimes(-u, b t)_{F} \otimes(a b(t+u),-a u(1+t))_{F} \\
& \simeq(-u, t)_{F} \otimes(a(t+u), u(1+t)(t+u))_{F} \otimes(b, 1+t)_{F} .
\end{aligned}
$$

Since $b$ is a uniformizing parameter for the $b$-adic valuation on $F$, it follows from 15. $\S 19.6$, Prop.] that the right side is a division algebra if (and only if) the algebra

$$
B=(-u, t)_{F_{1}((a))} \otimes(a(t+u), u(1+t)(t+u))_{F_{1}((a))} \otimes F_{1}(\sqrt{1+t})((a))
$$

is division. (Alternatively, one may view $A$ as a ring of twisted Laurent series over $B$ in an indeterminate whose square is $b$.) Now, $a(t+u)$ is a uniformizing parameter for the $a$-adic valuation on $F_{1}(\sqrt{1+t})((a))$, hence the same argument shows that $B$ is a division algebra if (and only if) the algebra

$$
C=(-u, t)_{F_{1}} \otimes F_{1}(\sqrt{1+t}, \sqrt{u(1+t)(t+u)})
$$

is division. Since $1+t$ and $u(t+u)$ are squares in $F_{0}(u)((t))$, we may embed $C$ in the quaternion algebra $(-u, t)_{F_{0}(u)((t))}$, which is clearly division. Therefore, $A$ is a division algebra.

\section{Non-totally Decomposable orthogonal inVolutions of DEgREe 8}

In this section, we consider the case of central simple algebras with orthogonal involution $(A, \sigma)$ of degree 8 that are not totally decomposable. These algebras do not contain any quaternion algebra with canonical involution $(H,-)$, since the centralizer of $H$ would be an algebra of degree 4 with symplectic involution, hence decomposable by [11, $(16.16)]$; the algebra $(A, \sigma)$ would then be totally decomposable.

We start with a couple of lemmas of independent interest related to triality. Let $Q_{1}, Q_{2}, Q_{3}$ be quaternion $F$-algebras such that $Q_{1} \otimes_{F} Q_{2} \otimes_{F} Q_{3}$ is split. By a well-known result due to Albert and to Pfister, this condition implies that $Q_{1}, Q_{2}$, 
and $Q_{3}$ have a common maximal subfield, see [11, (16.30)]. Therefore, we may write

$$
Q_{1}=\left(c, d_{1}\right)_{F}, \quad Q_{2}=\left(c, d_{2}\right)_{F}, \quad Q_{3}=\left(c, d_{3}\right)_{F}
$$

for some $c, d_{1}, d_{2}, d_{3} \in F^{\times}$such that the quaternion algebra $\left(c, d_{1} d_{2} d_{3}\right)_{F}$ is split. For $\alpha=1,2,3$, let $\rho_{\alpha}$ be the orthogonal involution on $Q_{\alpha}$ with $\operatorname{disc} \rho_{\alpha}=c$. The involution $\rho_{\alpha}$ is uniquely determined up to conjugation by $[11,(7.4)]$.

Lemma 6.1. For $\{\alpha, \beta, \gamma\}=\{1,2,3\}$ we have

$$
\left(Q_{\alpha},-\right) \otimes\left(Q_{\beta},-\right) \simeq \operatorname{Ad}_{\left\langle\left\langle d_{\alpha}\right\rangle\right\rangle} \otimes\left(Q_{\gamma}, \rho_{\gamma}\right) \simeq \operatorname{Ad}_{\left\langle\left\langle d_{\beta}\right\rangle\right\rangle} \otimes\left(Q_{\gamma}, \rho_{\gamma}\right)
$$

Proof. By Tao's computation of the Clifford algebra of a decomposable involution 17. we have

$$
\begin{aligned}
& C\left(\left(Q_{\alpha},-\right) \otimes\left(Q_{\beta},-\right)\right) \simeq Q_{\alpha} \times Q_{\beta} \simeq\left(c, d_{\alpha}\right)_{F} \times\left(c, d_{\beta}\right)_{F}, \\
& C\left(\operatorname{Ad}_{\left\langle\left\langle d_{\alpha}\right\rangle\right\rangle} \otimes\left(Q_{\gamma}, \rho_{\gamma}\right)\right) \simeq\left(c, d_{\alpha}\right)_{F} \times\left(c, d_{\alpha} d_{\gamma}\right)_{F} \simeq\left(c, d_{\alpha}\right)_{F} \times\left(c, d_{\beta}\right)_{F}, \\
& C\left(\operatorname{Ad}_{\left\langle\left\langle d_{\beta}\right\rangle\right\rangle} \otimes\left(Q_{\gamma}, \rho_{\gamma}\right)\right) \simeq\left(c, d_{\beta}\right)_{F} \times\left(c, d_{\beta} d_{\gamma}\right)_{F} \simeq\left(c, d_{\beta}\right)_{F} \times\left(c, d_{\alpha}\right)_{F} .
\end{aligned}
$$

Since central simple algebras with orthogonal involutions of degree 4 are classified by their Clifford algebra (see [11, (15.7)]), the lemma follows.

Now, for $\alpha=1,2,3$, let $\left(A_{\alpha}, \sigma_{\alpha}\right)$ be a central simple $F$-algebra with orthogonal involution of degree 8 such that for $\beta, \gamma$ with $\{\alpha, \beta, \gamma\}=\{1,2,3\}$,

$$
\left(A_{\alpha}, \sigma_{\alpha}\right) \simeq \operatorname{Ad}_{\left\langle 1,-1,1,-d_{\beta}\right\rangle} \otimes\left(Q_{\alpha}, \rho_{\alpha}\right) \simeq \operatorname{Ad}_{\left\langle 1,-1,1,-d_{\gamma}\right\rangle} \otimes\left(Q_{\alpha}, \rho_{\alpha}\right)
$$

Thus, $\left(A_{\alpha}, \sigma_{\alpha}\right)$ is Witt-equivalent to $\left(Q_{\beta},-\right) \otimes\left(Q_{\gamma},-\right)$ by Lemma 6.1.

Lemma 6.2. The triple $\left(\left(A_{1}, \sigma_{1}\right),\left(A_{2}, \sigma_{2}\right),\left(A_{3}, \sigma_{3}\right)\right)$ is trialitarian, in the sense that for $\{\alpha, \beta, \gamma\}=\{1,2,3\}$ we have

(See [11, p. 548].)

$$
C\left(A_{\alpha}, \sigma_{\alpha}\right) \simeq\left(A_{\beta}, \sigma_{\beta}\right) \otimes\left(A_{\gamma}, \sigma_{\gamma}\right)
$$

Proof. By triality, it suffices to prove the isomorphism for $\alpha=1, \beta=2$, and $\gamma=3$. By definition, $\left(A_{1}, \sigma_{1}\right)$ is an orthogonal sum of the algebra $M_{2}\left(Q_{1}\right)$ with a hyperbolic involution and of $\operatorname{Ad}_{\left\langle\left\langle d_{2}\right\rangle\right\rangle} \otimes\left(Q_{1}, \rho_{1}\right)$, so by Lemma 6.1

$$
\left(A_{1}, \sigma_{1}\right) \simeq\left(\left(M_{2}(F),-\right) \otimes\left(Q_{1},-\right)\right) \boxplus\left(\left(Q_{2},-\right) \otimes\left(Q_{3},-\right)\right) .
$$

By [11, (15.12)] we have

$$
C\left(\left(M_{2}(F),-\right) \otimes\left(Q_{1},-\right)\right) \simeq\left(M_{2}(F),-\right) \times\left(Q_{1},-\right)
$$

and

$$
C\left(\left(Q_{2},-\right) \otimes\left(Q_{3},-\right)\right) \simeq\left(Q_{2},-\right) \times\left(Q_{3},-\right)
$$

Arguing as in Garibaldi's "Orthogonal Sum Lemma" [6, Lemma 3.2], we get

$$
\left(C\left(A_{1}, \sigma_{1}\right), \underline{\sigma_{1}}\right) \simeq\left(C_{+}\left(A_{1}, \sigma_{1}\right), \sigma_{+}\right) \times\left(C_{-}\left(A_{1}, \sigma_{1}\right), \sigma_{-}\right)
$$

with

$$
\left(C_{+}\left(A_{1}, \sigma_{1}\right), \sigma_{+}\right) \simeq\left(\left(M_{2}(F),-\right) \otimes\left(Q_{2},-\right)\right) \boxplus\left(\left(Q_{1},-\right) \otimes\left(Q_{3},-\right)\right)
$$

and

$$
\left(C_{-}\left(A_{1}, \sigma_{1}\right), \sigma_{-}\right) \simeq\left(\left(M_{2}(F),-\right) \otimes\left(Q_{3},-\right)\right) \boxplus\left(\left(Q_{1},-\right) \otimes\left(Q_{2},-\right)\right) .
$$

Thus, $\left(C_{+}\left(A_{1}, \sigma_{1}\right), \sigma_{+}\right)$is Witt-equivalent to $\left(Q_{1},-\right) \otimes\left(Q_{3},-\right)$, hence it is isomorphic to $\left(A_{2}, \sigma_{2}\right)$. Likewise, $\left(C_{-}\left(A_{1}, \sigma_{1}\right), \sigma_{-}\right)$is isomorphic to $\left(A_{3}, \sigma_{3}\right)$. 
Theorem 6.3. Let $(A, \sigma)$ be a central simple $F$-algebra with orthogonal involution of degree 8. Assume $(A, \sigma)$ is not totally decomposable. Then $(A, \sigma)_{F_{Q}}$ is hyperbolic if and only if there is a quaternion $F$-algebra $Q^{\prime}$ with the following properties:

$-\operatorname{ind}\left(Q \otimes_{F} Q^{\prime}\right) \leq 2$, and

- $(A, \sigma)$ is Witt-equivalent to $(Q,-) \otimes\left(Q^{\prime},-\right)$.

When these equivalent properties hold, we can find $c, d, d^{\prime} \in F^{\times}$such that

$$
Q \simeq(c, d)_{F}, \quad Q^{\prime} \simeq\left(c, d^{\prime}\right)_{F}
$$

and

$$
(A, \sigma) \simeq \operatorname{Ad}_{\langle 1,-1,1,-d\rangle} \otimes\left(Q^{\prime \prime}, \rho^{\prime \prime}\right)
$$

where $Q^{\prime \prime}=\left(c, d d^{\prime}\right)_{F}$ and $\rho^{\prime \prime}$ is an orthogonal involution on $Q^{\prime \prime}$ with $\operatorname{disc} \rho^{\prime \prime}=c$.

Proof. Clearly, $(A, \sigma)_{F_{Q}}$ is hyperbolic if $(A, \sigma)$ is Witt-equivalent to an algebra containing $(Q,-)$. Conversely, suppose $(A, \sigma)_{F_{Q}}$ is hyperbolic. Since $(A, \sigma)$ is not totally decomposable, it is not hyperbolic. If $A$ is split, Proposition 2.1 shows that the anisotropic kernel of $(A, \sigma)$ is $\operatorname{Ad}_{n_{Q}} \simeq(Q,-) \otimes(Q,-)$, hence

$$
(A, \sigma) \simeq \operatorname{Ad}_{\langle 1,-1,1,-a\rangle} \otimes \operatorname{Ad}_{\langle\langle b\rangle\rangle} .
$$

For the rest of the proof, we may thus assume $A$ is not split. By Proposition 1.3, one of the components of the Clifford algebra, $C_{+}(A, \sigma)$ say, is split by $F_{Q}$. However, $C_{+}(A, \sigma)$ is not split since $(A, \sigma)$ is not totally decomposable, hence $C_{+}(A, \sigma)$ is Brauer-equivalent to $Q$. As was observed in Proposition 1.3, $\left(C_{+}(A, \sigma), \sigma_{+}\right)_{F_{Q}}$ is isotropic. If it is hyperbolic, then $(A, \sigma)$ is hyperbolic by the main theorem of [6], a contradiction. Therefore, the anisotropic kernel of $\left(C_{+}(A, \sigma), \sigma_{+}\right)$has degree 4 . It has discriminant 1 by triality, hence $\left(C_{+}(A, \sigma), \sigma_{+}\right)$is Witt-equivalent to a product $\left(Q^{\prime},-\right) \otimes\left(Q^{\prime \prime},-\right)$ for some quaternion $F$-algebras $Q^{\prime}, Q^{\prime \prime}$ such that $Q^{\prime} \otimes Q^{\prime \prime}$ is Brauer-equivalent to $Q$. We may therefore find $c, d, d^{\prime}, d^{\prime \prime} \in F^{\times}$such that

$$
Q \simeq(c, d)_{F}, \quad Q^{\prime} \simeq\left(c, d^{\prime}\right)_{F}, \quad Q^{\prime \prime} \simeq\left(c, d^{\prime \prime}\right)_{F} .
$$

Letting $\rho$ (resp. $\rho^{\prime}$, resp. $\rho^{\prime \prime}$ ) be an orthogonal involution on $Q$ (resp. $Q^{\prime}$, resp. $Q^{\prime \prime}$ ) with discriminant $c$, we have by Lemma 6.1

$$
\left(C_{+}(A, \sigma), \sigma_{+}\right) \simeq \operatorname{Ad}_{\left\langle 1,-1,1,-d^{\prime}\right\rangle} \otimes(Q, \rho) \simeq \operatorname{Ad}_{\left\langle 1,-1,1,-d^{\prime \prime}\right\rangle} \otimes(Q, \rho) .
$$

By Lemma 6.2, it follows that $(A, \sigma)$ is isomorphic to

$$
\operatorname{Ad}_{\langle 1,-1,1,-d\rangle} \otimes\left(Q^{\prime}, \rho^{\prime}\right) \quad \text { or } \quad \operatorname{Ad}_{\langle 1,-1,1,-d\rangle} \otimes\left(Q^{\prime \prime}, \rho^{\prime \prime}\right),
$$

hence it is Witt-equivalent to

$$
(Q,-) \otimes\left(Q^{\prime},-\right) \quad \text { or } \quad(Q,-) \otimes\left(Q^{\prime \prime},-\right) .
$$

Interchanging $Q^{\prime}$ and $Q^{\prime \prime}$ if necessary, we thus obtain the stated description of $(A, \sigma)$.

\section{Examples of ARBitrarily LARGE DEGREE}

Let $(A, \sigma)$ be a central simple $F$-algebra with involution of orthogonal or symplectic type. Consider the (iterated) Laurent series fields $F_{1}=F((x)), F_{2}=$ $F((x))((y))$, and the quaternion $F_{2}$-algebra $H=(x, y)_{F_{2}}$. Let $\rho$ be any involution of orthogonal or symplectic type on $H$, and let

$$
\left(A_{1}, \sigma_{1}\right)=(A, \sigma) \otimes_{F} \operatorname{Ad}_{\langle\langle x\rangle\rangle}, \quad\left(A_{2}, \sigma_{2}\right)=(A, \sigma) \otimes_{F}(H, \rho) .
$$


If $(A, \sigma)_{F_{Q}}$ is hyperbolic, then $\left(A_{1}, \sigma_{1}\right)_{F_{Q}}$ and $\left(A_{2}, \sigma_{2}\right)_{F_{Q}}$ also are hyperbolic, since they contain a hyperbolic factor.

Theorem 7.1. Assume $(A, \sigma)$ is anisotropic. Then $\left(A_{1}, \sigma_{1}\right)$ and $\left(A_{2}, \sigma_{2}\right)$ are anisotropic. Moreover, the following conditions are equivalent:

(i) $(A, \sigma)$ contains $(Q,-)$;

(ii) $\left(A_{1}, \sigma_{1}\right)$ contains $(Q,-)$;

(iii) $\left(A_{2}, \sigma_{2}\right)$ contains $(Q,-)$.

Proof. Let $\xi_{1}=\left(\begin{array}{cc}1 & 0 \\ 0 & -1\end{array}\right), \eta_{1}=\left(\begin{array}{ll}0 & x \\ 1 & 0\end{array}\right) \in M_{2}\left(F_{1}\right)$, so

$$
\operatorname{ad}_{\langle\langle x\rangle\rangle}\left(\xi_{1}\right)=\xi_{1} \quad \text { and } \quad \operatorname{ad}_{\langle\langle x\rangle\rangle}\left(\eta_{1}\right)=-\eta_{1} \text {. }
$$

Let also $\left(a_{i}\right)_{i \in I}$ be an $F$-basis of $A$, so $\left(a_{i} \otimes 1, a_{i} \otimes \xi_{1}, a_{i} \otimes \eta_{1}, a_{i} \otimes \xi_{1} \eta_{1}\right)_{i \in I}$ is an $F_{1}$-basis of $A_{1}$. We extend the $x$-adic valuation $v_{1}$ on $F_{1}$ to a map

defined by

$$
g_{1}: A_{1} \rightarrow\left(\frac{1}{2} \mathbb{Z}\right) \cup\{\infty\}
$$

$g_{1}\left(\sum_{i \in I} a_{i} \otimes\left(\alpha_{i}+\beta_{i} \xi_{1}+\gamma_{i} \eta_{1}+\delta_{i} \xi_{1} \eta_{1}\right)\right)=\min _{i \in I}\left(v_{1}\left(\alpha_{i}\right), v_{1}\left(\beta_{i}\right), v_{1}\left(\gamma_{i}\right)+\frac{1}{2}, v_{1}\left(\delta_{i}\right)+\frac{1}{2}\right)$

for $\alpha_{i}, \beta_{i}, \gamma_{i}, \delta_{i} \in F_{1}$. It is readily verified that the map $g_{1}$ satisfies the following conditions for $s, t \in A_{1}$ and $\alpha \in F_{1}$ :

- $g_{1}(1)=0$ and $g_{1}(s)=\infty$ if and only if $s=0$;

- $g_{1}(s+t) \geq \min \left(g_{1}(s), g_{1}(t)\right)$ and $g_{1}(s \alpha)=g_{1}(s)+v_{1}(\alpha)$;

- $g_{1}(s t) \geq g_{1}(s)+g_{1}(t)$.

(It suffices to prove the last inequality for $s, t$ in the above $F_{1}$-base of $A_{1}$, see [20. Lemma 1.2].) The map $g_{1}$ defines a filtration of $A_{1}$, and the associated graded ring $\operatorname{gr}\left(A_{1}\right)$ is

with the grading defined by

$$
\operatorname{gr}\left(A_{1}\right) \simeq A \otimes_{F} M_{2}\left(F\left[x, x^{-1}\right]\right)
$$

$$
\begin{array}{ll}
\operatorname{gr}\left(A_{1}\right)_{\lambda}=A \otimes\left(\begin{array}{cc}
x^{\lambda} & 0 \\
0 & x^{\lambda}
\end{array}\right) & \text { for } \lambda \in \mathbb{Z} \\
\operatorname{gr}\left(A_{1}\right)_{\lambda}=A \otimes\left(\begin{array}{cc}
0 & x^{\lambda+\frac{1}{2}} \\
x^{\lambda-\frac{1}{2}} & 0
\end{array}\right) & \text { for } \lambda \in\left(\frac{1}{2} \mathbb{Z}\right) \backslash \mathbb{Z} .
\end{array}
$$

Therefore, $\operatorname{gr}\left(A_{1}\right)$ is a graded simple algebra, and $g_{1}$ is a $v_{1}$-gauge in the sense of 20. The involution $\sigma_{1}$ preserves $g_{1}$. On $\operatorname{gr}\left(A_{1}\right)_{0}$, the induced involution $\widetilde{\sigma_{1}}$ is $\sigma \otimes \mathrm{Id}$, hence it is anisotropic. Therefore, $\sigma_{1}$ is anisotropic by [21, Cor. 2.3], $g_{1}$ is the unique $v_{1}$-gauge that is preserved by $\sigma_{1}$ by [21, Th. 2.2], and we have

$$
g_{1}\left(\sigma_{1}(s) s\right)=2 g_{1}(s) \quad \text { for all } s \in A_{1} \text {. }
$$

Now, suppose $\left(A_{1}, \sigma_{1}\right)$ contains $(Q,-)$; it then contains elements $i, j$ such that

$$
i^{2}=a, \quad j^{2}=b, \quad j i=-i j, \quad \sigma_{1}(i)=-i, \quad \sigma_{1}(j)=-j .
$$

Then by (14) we have $g_{1}(i)=\frac{1}{2} g_{1}(-a)=0$ and, similarly, $g_{1}(j)=0$. The images $\widetilde{i}, \widetilde{j}$ of $i, j$ in $\operatorname{gr}_{1}\left(A_{1}\right)_{0}$ satisfy conditions similar to (15). Since $\operatorname{gr}\left(A_{1}\right)_{0} \simeq A \times A$ we may consider a projection $\operatorname{gr}(A)_{0} \rightarrow A$, which is a homomorphism of algebras with involution $\pi:\left(\operatorname{gr}\left(A_{1}\right)_{0}, \widetilde{\sigma_{1}}\right) \rightarrow(A, \sigma)$. The images $\pi(\widetilde{i}), \pi(\widetilde{j})$ generate a copy of $(Q,-)$ in $(A, \sigma)$. Thus, $(A, \sigma)$ contains $(Q,-)$ if $\left(A_{1}, \sigma_{1}\right)$ contains $(Q,-)$. The converse is clear. 
The argument for $\left(A_{2}, \sigma_{2}\right)$ follows the same lines. Let $\xi_{2}, \eta_{2} \in H$ be such that

$$
\xi_{2}^{2}=x, \quad \eta_{2}^{2}=y, \quad \eta_{2} \xi_{2}=-\xi_{2} \eta_{2} .
$$

Note that if $\rho$ is orthogonal its discriminant is represented by the quadratic form $\langle x, y,-x y\rangle$, hence it is the square class of $x, y$, or $-x y$. Therefore, we may assume $\rho=\operatorname{Int}\left(\xi_{2}\right) \circ-, \operatorname{Int}\left(\eta_{2}\right) \circ-$, or $\operatorname{Int}\left(\xi_{2} \eta_{2}\right) \circ-$. In each case (and also if $\rho$ is symplectic) we have $\rho\left(\xi_{2}\right)= \pm \xi_{2}$ and $\rho\left(\eta_{2}\right)= \pm \eta_{2}$.

Let $v_{2}: F_{2} \rightarrow \mathbb{Z}^{2} \cup\{\infty\}$ be the $(x, y)$-adic valuation such that $v_{2}\left(x^{\lambda} y^{\mu}\right)=(\lambda, \mu)$ for $\lambda, \mu \in \mathbb{Z}$, where $\mathbb{Z}^{2}$ is endowed with the right-to-left lexicographic ordering. Considering again an $F$-basis $\left(a_{i}\right)_{i \in I}$ of $A$, we extend $v_{2}$ to a map

$$
g_{2}: A_{2} \rightarrow\left(\frac{1}{2} \mathbb{Z}\right)^{2} \cup\{\infty\}
$$

defined by

$$
\begin{aligned}
& g_{2}\left(\sum_{i \in I} a_{i} \otimes\left(\alpha_{i}+\beta_{i} \xi_{2}+\gamma_{i} \eta_{2}+\delta_{i} \xi_{2} \eta_{2}\right)\right)= \\
& \min _{i \in I}\left(v_{2}\left(\alpha_{i}\right), v_{2}\left(\beta_{i}\right)+\left(\frac{1}{2}, 0\right), v_{2}\left(\gamma_{i}\right)+\left(0, \frac{1}{2}\right), v_{2}\left(\delta_{i}\right)+\left(\frac{1}{2}, \frac{1}{2}\right)\right)
\end{aligned}
$$

for $\alpha_{i}, \beta_{i}, \gamma_{i}, \delta_{i} \in F_{2}$. The map $g_{2}$ is a $v_{2}$-gauge on $A_{2}$ with associated graded ring

$$
\operatorname{gr}\left(A_{2}\right)=A \otimes(x, y)_{F\left[x, x^{-1}, y, y^{-1}\right]} .
$$

The involution $\sigma_{2}$ preserves $g_{2}$ and the induced involution $\widetilde{\sigma_{2}}$ on $\operatorname{gr}\left(A_{2}\right)_{0}=A$ is $\sigma$. Therefore, the same arguments as for $\left(A_{1}, \sigma_{1}\right)$ show that $\left(A_{2}, \sigma_{2}\right)$ is anisotropic, and that $\left(A_{2}, \sigma_{2}\right)$ contains $(Q,-)$ if and only if $(A, \sigma)$ contains $(Q,-)$.

Theorem 7.1 applies in particular to the division algebra with orthogonal involution $(A, \sigma)$ of Theorem 5.2, and yields central simple algebras with anisotropic involution $\left(A_{1}, \sigma_{1}\right)$ and $\left(A_{2}, \sigma_{2}\right)$ of degree 16 that do not contain $(Q,-)$, even though they are hyperbolic over $F_{Q}$. The involution $\sigma_{1}$ is orthogonal and ind $A_{1}=8$, while the involution $\sigma_{2}$ may be of orthogonal or symplectic type and $A_{2}$ is division. Of course, these constructions can be iterated to obtain examples of algebras with anisotropic involution of arbitrarily large degree that become hyperbolic over $F_{Q}$ and do not contain $(Q,-)$. Such examples can also be derived from the central simple algebras of degree 4 with symplectic involution in case (b) of Theorem 3.6. although no division algebra can be obtained in this way since the algebras in case (b) of Theorem 3.6 have index 2.

\section{REFERENCES}

[1] J. Kr. Arason, Cohomologische Invarianten quadratischer Formen, J. Alg. 36 (1975), 448491.

[2] E. Bayer-Fluckiger, D. Shapiro, and J.-P. Tignol, Hyperbolic involutions, Math. Z. 214 (1993), no. 3, 461-476.

[3] E. Bayer-Fluckiger, R. Parimala, and A. Quéguiner-Mathieu, Pfister involutions, Proc. Indian Acad. Sci. Math. Sci. 113 (2003), no. 4, 365-377.

[4] K.J. Becher, A proof of the Pfister Factor Conjecture, Invent. Math. (2008), [DOI 10.1007/s00222-007-0107-5].

[5] I. Dejaiffe, Formes antihermitiennes devenant hyperboliques sur un corps de déploiement, C.R. Acad. Sci. Paris, Série I 332 (2001), no. 2, 105-108.

[6] R.S. Garibaldi, Clifford algebras of hyperbolic involutions, Math. Z. 236 (2001), 321-349, [DOI 10.1007/s002090000180].

[7] - Orthogonal involutions on algebras of degree 16 and the Killing form of $E_{8}$ (with an appendix by K. Zainoulline), preprint. 
[8] D.W. Hoffmann, Function fields of quadratic forms, Doctoral dissertation. University of California, Berkeley, California 1992.

[9] D.W. Hoffmann, D.W. Lewis, and J. Van Geel, Minimal forms for function fields of conics, $K$-theory and algebraic geometry: connections with quadratic forms and division algebras (Santa Barbara, CA, 1992), Proc. Sympos. Pure Math., vol. 58, Amer. Math. Soc., Providence, RI, 1995, pp. 227-237.

[10] D.W. Hoffmann and J. Van Geel, Minimal forms with respect to function fields of conics, Manuscripta Math. 86 (1995), no. 1, 23-48.

[11] M.-A. Knus, A.S. Merkurjev, M. Rost, and J.-P. Tignol, The book of involutions, Colloquium Publ., vol. 44, Amer. Math. Soc., Providence, RI, 1998.

[12] T.Y. Lam, Introduction to quadratic forms over fields, Amer. Math. Soc., Providence, RI, 2005.

[13] A.S. Merkurjev, Simple algebras and quadratic forms, Izv. Akad. Nauk SSSR Ser. Mat. 55 (1992), no. 1, 218-224, English translation : Math. USSR-Izv. 38 (1992), no. 1, 215-221.

[14] R. Parimala, R. Sridharan, and V. Suresh, Hermitian analogue of a theorem of Springer, J. Algebra 243 (2001), 780-789.

[15] R.S. Pierce, Associative algebras, Springer, New York, 1982.

[16] W. Scharlau, Quadratic and Hermitian forms, Springer, Berlin, 1985.

[17] D. Tao, A variety associated to an algebra with involution, J. Algebra 168 (1994), no. 2, 479-520.

[18] J.-P. Tignol, Réduction de l'indice d'une algèbre simple centrale sur le corps des fonctions d'une quadrique, Bull. Soc. Math. Belg. Sér. A 42 (1990), no. 3, 735-745.

[19], A Cassels-Pfister theorem for involutions on central simple algebras, J. Algebra 181 (1996), 857-875.

[20] J.-P. Tignol, and A.R. Wadsworth, Value functions and associated graded rings for semisimple algebras, to appear in Trans. Amer. Math. Soc.

[21] Valuations on algebras with involution, in preparation.

Université Paris 13 (LAGA), CNRS (UMR 7539), Université Paris 12 (IUFM), 93430 Villetaneuse, France

E-mail address: queguin@math.univ-paris13.fr

$U R L:$ http://www-math.univ-paris13.fr/ queguin/

Département de Mathématiques, Université Catholique de Louvain, Chemin du CyClotron, 2, B1348 Louvain-la-Neuve, Belgique

E-mail address: jean-pierre.tignol@uclouvain.be

URL: http://wwww.math.ucl.ac.be/membres/tignol 